\title{
评 述激光先进制造专题
}

\section{多能场微射流水导激光加工研究发展概况}

\author{
卢希㻇 ${ }^{1,2,3^{*}}$, 江开勇 ${ }^{1,3}$ \\ 1. 华侨大学机电及自动化学院, 厦门 361021 ; \\ 2. 厦门大学电子科学与技术学院, 厦门 361005 ; \\ 3. 华侨大学福建省特种能场制造重点实验室, 厦门 361021 \\ *联系人, E-mail: luxizhao@hqu.edu.cn
}

收稿日期: 2019-05-27; 接受日期: 2019-07-25; 网络出版日期: 2020-01-07

国家自然科学基金(编号: 51575198)和福建省重点实验室开放基金(编号: 2016JZA002)资助项目

\begin{abstract}
摘要 微射流水导激光加工属于一种多能场复合激光加工技术. 该技术利用加工激光耦合稳定压力微射流, 改变 激光能量分布为平顶高斯光束, 并对加工断面进行冷却、冲刷. 稳定的小流量压力去离子水射流导引激光柔性延 伸加工焦点, 改善加工激光能量分布的同时实现去屑、冷却, 使得其轴向加工质量、精度得到提高, 并提高激光 轴向加工能力. 本文介绍并阐述了该加工装置及设备研发的若干关键因素: 激光全反射入射角度的选择、光束质 量、模式、聚焦位置及激光与水射流的耦合精度. 对比了该技术与超短脉冲激光(飞秒激光)的加工特点. 阐述了 微射流水导激光适当控制激光加工热量, 控制激光加工方向, 适用于高质量大深径比(约 10-100)的深孔、槽、缝 的场合以及一些低 $k$ 绝缘材质, 硬质合金、 $\mathrm{SiC} 、 \mathrm{GaAs}$ 、聚晶金刚石等材质的高附加值对象场合.
\end{abstract}

关键词 微射流水导激光加工, 耦合, 全反射传输, 加工质量

PACS: 42.30.Sy, 42.62.Be, 47.15.Fe, 47.15.Gf

\section{1 引言}

众所周知，短脉冲及传统激光加工质量及精度受 限于激光作用周边区域的热影响以及激光光束焦斑质 量的影响, 因而难以提高. 1993年, 瑞士Richerzhagen等

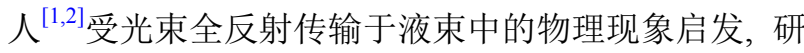
发出相关装置及设备并申请微射流水导激光专利, 并 在Lausanne成立Synova公司开发推广相关应用。本文 讨论的主要对象为微射流水导激光(Laser Microjet, LMJ), 亦称水导激光(Water Jet Guided Laser) ${ }^{[3-5]}$, 是
一种加工微米尺度的高效深度水射流“同轴”全反射导 引激光复合加工方式, 该加工方式结合高能激光加工 和微水射流加工的部分特点及优点，属于柔性的激光 能场和水射流微加工方式的复合. 微射流水导激光区 别于高压(约>30 MPa)、超高压纯水射流喷射切割而 伴随的激光只起软化、改性表面组织作用的加工方 法，或者激光作用于加工面的边侧同时侧向复合水射 流冲刷的多能场加工方式. 激光耦合去离子水射流技 术工作原理如图1所示，毫秒(ms)-飞秒(fs)激光耦合传 输于稳定微水射流中 ${ }^{[6]}$, 通过稳定微水射流的柔性延

引用格式: 卢希钊, 江开勇. 多能场微射流水导激光加工研究发展概况. 中国科学: 物理学 力学 天文学, 2020, 50: 034203 Lu X Z, Jiang K Y. Research and application development of compound energy field processing - laser microjet (in Chinese). Sci Sin-Phys Mech Astron, 2020, 50: 034203, doi: 10.1360/SSPMA-2019-0194 


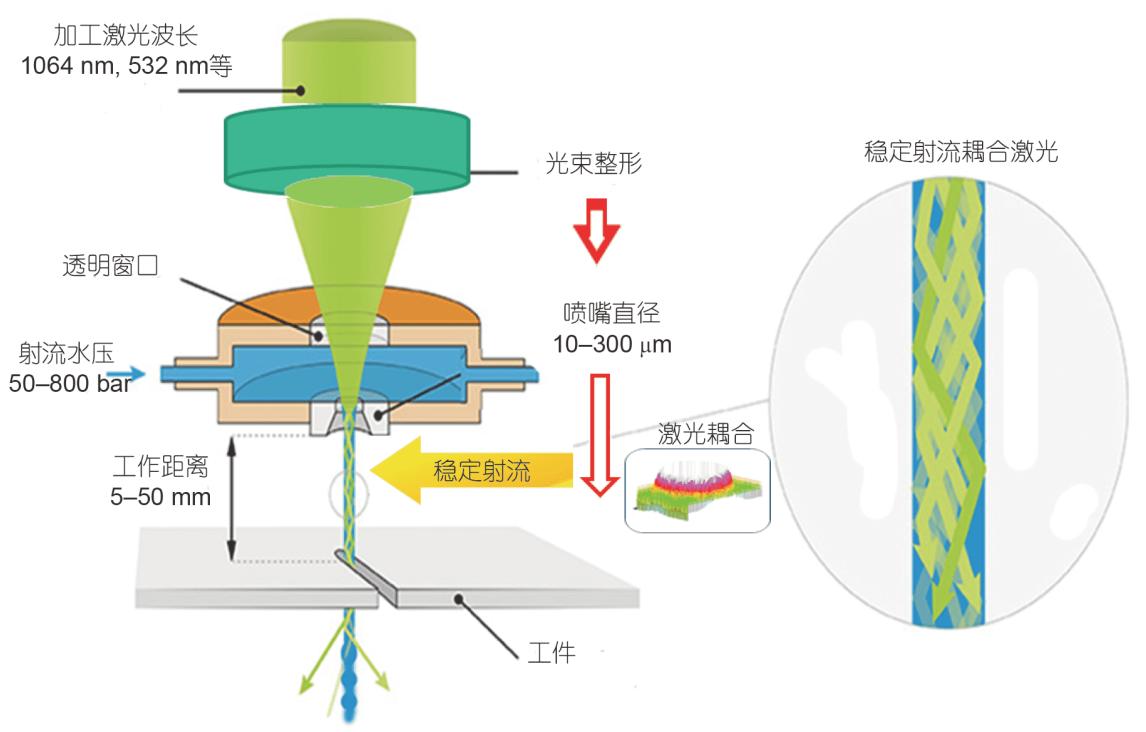

图 1 (网络版彩图)微射流水导激光工作原理图

Figure 1 (Color online) Principle scheme of laser microjet.

伸, 实现加工激光于水束光纤的全反射传输. 该技术耦 合不同激光器可以用于加工 $10 \mu \mathrm{m}$ 尺度的, 边缘要求较 高的场合. 目前, 该方式可加工大约 5-25 mm以上厚度 的工件 ${ }^{[7]}$, 包括晶圆、太阳能硅光板的划片、切割、 划槽, 飞机涡轮叶片的气膜孔打孔等边缘要求较高的 应用场合，主要涉及的加工对象包括不同硬度、不同 反射率的材料 ${ }^{[8]}$, 如不锈钢, $\mathrm{Ni}-\mathrm{Ti}$ 等金属; 硬质合金, 如立方氮化嗍、聚晶金刚石、碳化铇等 ${ }^{[9]}$; 半导体、 光电、超硬材料, 如薄硅片 ${ }^{[10,11]} 、 D^{2}$ iamond $d^{[12] 、}$ $\mathrm{GaAs}^{[13]}$ 、InP等微细加工、绿色制造及医疗高附加值 的零、部件 ${ }^{[14]}$.

微射流水导激光有别于传统纯水射流加工通过高 压或超高压水冲击破碎加工，该加工方式的水射流主 要作用是传输加工激光至被加工表面, 同时将熔融多 余热量及残渣带走. 其加工的物理机理是激光照射被 加工表面，该表面吸收一定频率的光子能量(以毫秒 (ms)-纳秒(ns)激光为例, 波长越长, 光子能量越小), 改 变被加工表面的自由电子运动状态, 使得其原子或分 子的存在状态发生变化, 即固态转换成气态甚至离子 态, 并脱离物质本体 ${ }^{[15]}$. 在此变化过程中, 物质内的原 子或分子通过热的形式向加工区域周围释放一定的能 量, 不同波长、脉宽的激光加工实质是激光光波(光 子)作用于材料, 材料吸收光波(光子), 由于光子能量 及吸收效果不同, 故而产生有差异的光热效应, 使得
加工区域形成的热量从内部传导到外部，加工区域的 温度显著提高, 此热量作用材料表面的主要效果为去 除加工相变区域, 也把多余的热量传递给周边区域, 形成热影响区(Heat Affected Zone, HAZ), 导致重铸 层、微裂纹、毛刺、熔渣等瑕疪, 使得激光精密加工 无法继续提升加工质量及精度, 微射流水导激光则为 传导多余加工热和控制热提供新的方案.

正如图1所示, 稳定的微射流在击穿阈值内可耦合 不同波长、脉宽等参数的激光, 加工作用于不同材质 的工件, 从而通过不同的水射流参数选取, 可以影响 水导激光的加工效率和加工质量, 提高激光加工盲孔/ 通孔的深度, 改善加工对象表面的质量, 提高加工立面 的垂直度和内壁质量.

\section{2 多能场微射流水导激光加工技术特点}

微射流水导激光结合了传统激光、短脉冲激光以 及水射流冲击的特点及优点, 耦合激光能场、射流场 作用于待加工工件表面, 控制加工过程中产生的热量 及多余能量, 从而达到提高加工质量和精度的目的.

\section{1 微射流水导激光具备传统激光以及短脉冲激 光的优点和特点}

微射流水导激光加工本质上仍属于传统激光加 
工，对加工激光参数的选择基于待加工材料对不同波 长、不同脉宽激光的吸收效率、加工阈值，同时考虑 加工激光对微水射流的穿透性、激光的横模模式、耦 合角度 ${ }^{[16,17]}$ ，避免因水射流的不同入射角度、同轴 度、射流稳定压力、散热效率，激光到达待加工面的 效率降低, 冲刷熔渣(气化残渣颗粒约 $1 \mu \mathrm{m}^{[8]}$ )从而影 响短脉冲激光加工效果.

(1) 微射流水导激光加工可以低损耗地传输于稳 定射流. 微射流水导激光加工耦合时须保证一定的入 射角度并运送加工能量于水射流中．其加工时，产生 的冲击小、机械应力小、激光相对于机加工的刃具损 耗小, 可以几乎无损耗地进行重复加工, 并具有优良的 加工截交面、前立面(相当于机械刀具中的前刃角), 同时可以大幅减轻激光加工的雉角 ${ }^{[2,8]}$, 克服了占较多 可燃成分(氧气)的空气气体对激光热加工的干扰.

(2) 微射流水导激光加工方式可在一定范围内柔 性延伸激光加工的焦点, 使得在一定的激光传输距离 范围内可以不考虑激光焦点的具体位置，操作简便， 同时也可在加工范围内加工出一定长径比的深槽或 深孔.

(3) 微射流水导激光加工可在一定范围内减少热 效应，控制热影响区范围. 微射流水导激光射流的流 动可以带走加工时传递的热量，通过水射流控制激光 加工堆积热, 可以减小甚至消除热影响区和热裂纹, 并在一定程度上消除激光加工边缘的重铸层、毛刺、 热裂的影响 ${ }^{[8]}$.

(4) 微射流水导激光加工可在加工距离内提高其 沿激光传递的轴(纵)向加工效率. 微射流水导激光改 变激光在约束的水射流内部的径向及轴向能量分布, 同时也使得激光轴向加工能力获得比径向更大的提 高，且这改变了传统激光加工方向，同偏振光的加 工 $^{[17]}$ 有异曲同工之妙.

(5) 微射流水导激光加工可降低加工过程的粉尘 污染，提升加工断面的粗糙度及洁净度. 工作过程中, 射流冲刷作用以及其他作用力，可及时将凝固的加工 残渣、粉尘、颗粒带走, 从而降低加工污染 ${ }^{[18,19]}$.

(6) 微射流水导激光加工可以提升切割和加工的 厚度. 此时，射流的冲刷作用可以将残渣、熔渣、颗 粒、毛刺带离加工面，以此提高加工侧端面的质量， 使得射流传输速度一致，从而降低拉曼散射影响，有 利于进一步提高加工深度.
该多能场耦合加工方法中的射流场所起的作用包 括冲刷残渣，加速凝固激光液化的残渣、粉尘、颗粒 等，并控制方向被耦合射流带走．射流自喷嘴出射一 段距离后，其对于所加工的壁沿产生冲击作用，射流 达到加工面时，由于冲击作用，产生的反作用力驱动 射流把加工的熔渣、粉尘、颗粒、残渣带离加工表 面; 根据大量实验表明, 高压同轴的去离子水射流可以 更高效地带走加工区域发生相态变化的粉尘、颗粒、 蒸气(对激光的穿透加工仍然有较大影响), 但是压力 过高，较大射流入射角度，也使得水射流在进入喷嘴 前压力变化过于剧烈，因此水射流过早地散开，乃至 喷溅(喷嘴加工工作表面质量不高时). 此时, 激光加工 的冷却效率更高，使其无法继续上升且影响脉冲激光 的下一序列脉冲的加工. 另外, 以一定压力稳定前进 的水射流同时影响加工区域的热堆积，并稳定地带走 加工后所形成的残渣及热量, 即为微射流水导激光找 到一个可以不断更新的待加工断面.

当微射流水导激光加工时，持续吸收光子的各分 子/原子产生电子振荡, 碰撞产生的热量. 如此, 可以降 低激光水浴加工温度; 同时对水导激光加工的被加工 面进行退火、淬火热处理，使得金相组织得到改善; 同时同轴水射流可以冲刷加工后的残渣离开加工区 域, 激光复合水射流加工, 激光诱导等离子体水中辅助 加工方式, 该加工方式的特点是激光牺牲部分激光峰 值功率, 穿过水层, 改变激光能量分布, 同时, 由于稳 定水射流能冲刷带走加工的熔渣，而且随着加工深度 的延伸, 焦深更短的激光在理论上传输效率能得到更 明显的改善. 微射流水导激光的关键技术包含稳定压 力水射流的产生和激光与水射流光纤的耦合.

国内外相关课题组对激光的模式、耦合入射角 度、耦合点(射流径向、轴向误差及入射角度误差)进 行相应仿真, 并使用能量计及光束分析仪分析了激光 传输的质量及效率 ${ }^{[8,20]}$, 能量分布变化情况; 并对射流 在加工中的散热作用以及射流对加工激光的传输效率 做了大量仿真 ${ }^{[21-24]}$.

\section{2 微射流水导激光和飞秒激光加工应用场合}

飞秒激光则是另一类典型的“冷加工”[14]，具有超 高的脉冲功率峰值, 该特点决定其加工区别于常规激 光, 故而也就同传统微射流水导激光加工有本质区别. 飞秒激光的单脉冲激光作用时间远小于晶格振荡、原 
子相互挤压碰撞的皮秒 $(\mathrm{ps})$ 级时间. 在加工上，控制良 好的微秒 $(\mu \mathrm{s})$ 级微射流水导激光同飞秒激光加工相比, 仍是传统激光加工，两者加工的效果差别如图2所示. 飞秒激光加工时基本无固态液相的变化，显示激光诱 导周期表面结构(Laser Induced Periods Surface Structures, LIPSS)的特征, 甚至无重铸层 ${ }^{[6]}$; 微射流水导激 光与飞秒激光加工相比, 其加工厚度具有优势, 范围更 大; 微射流水导激光加工范围可以达到约 5-25 mm, 而 典型的飞秒激光加工深度仅为约 $1 \mathrm{~mm}$. 此外，对某些 材料的溶液可以实现绿色加工, 如GaAs的加工中, 由 于As离子的毒性及其易溶于水的特点，可利用水射流 带走含As的加工残渣，实现可控污染的绿色加工．因 此, 微射流水导激光加工满足各种加工厚度要求，工 业应用适应性更好.

此外, 飞秒激光加工通常应用于实验室和洁净空 间，其对于激光设备的电源支持和控制要求更高；对 加工所处空间的温度、洁净度、加工噪声以及加工环 境的温度、粉尘控制等要求更严格; 而微射流水导激 光则由于多能场复合，对加工射流工作条件较高，但 对于加工激光的电源支持要求较为宽泛，对于加工环 境也具备较高的适应性，可以应用于传统工业背景的 更加恶劣的工作环境.

以典型的微秒级微射流水导激光加工的质量与 $800 \mathrm{fs}$ 飞秒激光加工为例, 短脉冲激光微射流水导激光 的加工激光参数如表 1 所示.

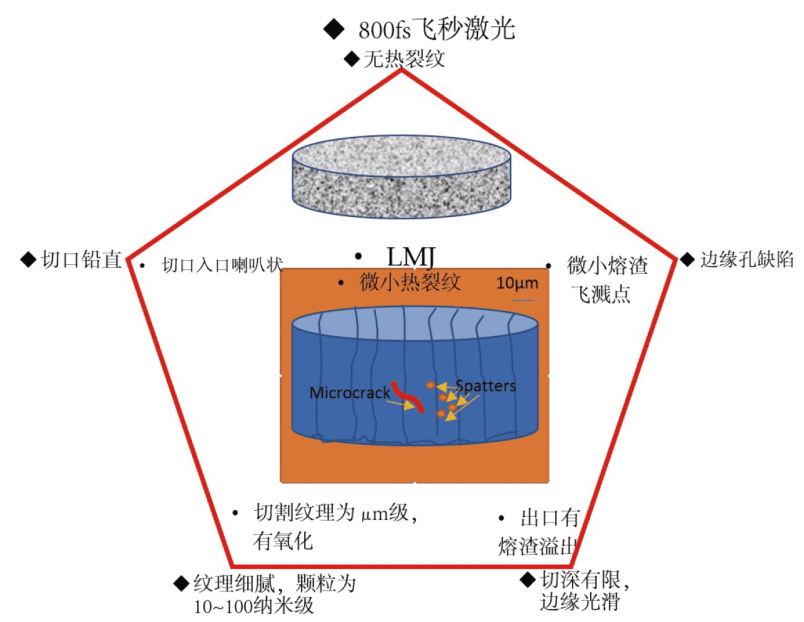

图 2 (网络版彩图)典型微射流水导激光与飞秒激光加工质 量比较 ${ }^{[6]}$

Figure 2 (Color online) Comparison of the quality of drilling holes between laser microjet and $800 \mathrm{fs}$ femtosecond laser [6].
表 1 微射流水导激光与加工飞秒激光参数

Table 1 Laser parameters of LMJ and femtosecond laser

\begin{tabular}{ccc}
\hline 参数 & 微射流水导激光 & 飞秒激光 \\
\hline Wavelength $(\mathrm{nm})$ & 1064 & 1552 \\
Pulse duration & $20 \mu \mathrm{s}$ & $800 \mathrm{fs}$ \\
Pulse energy & $9 \mathrm{~mJ}$ & $50 \mu \mathrm{J}$ \\
Fluence $\left(\mathrm{J} / \mathrm{cm}^{2}\right)$ & 2.866 & - \\
Peak power $(\mathrm{W}$ peak) & 450 & - \\
Pulse repetition frequency & 2000 & 100000 \\
$(\mathrm{~Hz})$ & - & 75 \\
Tangential speed $(\mathrm{mm} / \mathrm{s})$ & 5.7 & - \\
Specific power $\left(\mathrm{MW} / \mathrm{cm}^{2}\right)$ & - & 20 \\
Spot diameter $(\mu \mathrm{m})$ & &
\end{tabular}

University of Pisa的Romolia等人 ${ }^{[6]}$ 进行了表1中 $20 \mu \mathrm{s}$ 的微射流水导激光与 $800 \mathrm{fs}$ 激光深度加工断面的 质量比较, 两者光束整合应用的都是场镜进行聚焦. 微 射流全反射 $\mu \mathrm{s}$ 级激光, 其加工边缘仍可能出现微裂纹, 出现一些附带熔融状态的延伸物; $800 \mathrm{fs}$ 级激光在有效 加工深度范围内进行钻孔, 加工机理上并未出现热堆 积、热扩散, 故而加工边缘出现细小的纳米颗粒, 组 织细淢达到纳米级别, 边缘较为整齐光滑, 热效应也 基本得到控制.

图2中五边形内部为微射流水导加工的特点, 五边 形外部则为 $800 \mathrm{fs}$ 激光加工的一些对应优点. 在有效加 工轴向厚度内，微射流水导激光加工虽可以改善常规 激光加工的热扩散, 加工深度和厚度更深, 热堆积等 热影响区得到控制, 但仍有微小熔渣和飞溅点, 出口 锥度也较大呈喇叭状, 切割的组织纹理为 $\mu \mathrm{m}$ 级的条纹 状; 而飞秒激光由于散热机理本质上不同, 加工质量更 高, 更适合雉度要求更高的微纳加工, 且无热裂纹 出现.

\section{3 微水射流导引激光耦合技术}

微射流水导激光加工技术的核心装置是强激光与 稳定微射流的耦合装置. 该耦合装置可分为激光与水 射流的耦合光路部分和稳定射流发生装置. 该装置的 设计影响加工激光经微水射流导引进入喷嘴的耦合效 果, 进而影响激光传输的效率.

激光入射射流之前, 需要对激光光束重新聚焦, 调 
整入射角度, 聚焦焦点使得激光和射流同轴, 同轴性越 好, 激光传输效率越高, 获得的可传导激光效果越好. 国内外在耦合加工激光进入喷嘴的技术主要采用轴棱 雉无衍射光提升聚焦分辨率 ${ }^{[25]}$, 倒置望远镜可靠聚焦 进入喷嘴，径向、轴向上分别用透镜在各自方向上微 位移以满足径向、轴向的精准对焦(各轴精度约为 $2 \mu \mathrm{m})^{[26]}$.

激光传输在水射流光纤中自光密介质进入光疏介 质, 会产生全反射现象, 存在全反射临界角, 即当入射 角度大于全反射临界角度时, 全反射现象将会消失. 激 光通过全反射临界角向前传输，当入射角度大于全反 射临界角度时，水射流内的加工激光全反射传输现象 将会消失. 实际上, 加工激光入射角度增大, 使得激光 加工的尺寸更大, 且激光加工能量分布更加均匀. 而在 考察入射角变化对反射光斑亮度影响的实验中，从光 束分析仪器中可以看到光斑中的光束明暗分布更精 细, 条纹间隔更细小, 反射光的亮度随入射角增大而减 小; 若入射角度减小则出射待加工光斑变小.

微射流水导激光技术主要涉及激光的光束整形、 激光同稳定水射流的耦合装置设计.

光束整形技术可以通过分散后再聚焦, 通过对应 的透镜组合、倒置望远镜组、轴雉棱镜等方法进行对
准耦合, 选择工作合适的洜(一般选择柱塞洜以保证射 流可以持续稳定工作), 水射流的腔体水层厚度主要涉 及激光耦合的入射角度、焦深长度以及微水射流的稳 定性; 激光耦合装置的设计影响激光的耦合能否精确 入射喷嘴以及传输多模激光的效率, 改善加工激光分 布; 该设计也影响激光耦合水束光纤之前的损耗，激 光耦合进入腔体, 以及喷嘴的实际可维护性.

各耦合装置都有各自的能量密度和观测要求. 其 中工业化应用较成熟、完善的是不断推陈出新的图3 (a)和(b)的Synova公司主页上的产品, 国内也有一些该 装置研发见诸报道. 如可适应一定范围光功率的激光 聚焦要求的轴雉棱镜应用 ${ }^{[25]}$, 图3(e)为清华大学的三 轴离轴调整装置 ${ }^{[26]}$, 可调的各轴精度可以达到 $2 \mu \mathrm{m}$. 关于整体耦合装置的结构则有相关仿真和设计 ${ }^{[27,28]}$, 为改善散热, 选铜为腔体材料 ${ }^{[29]}$. 聚焦设计中也有采 用倒置望远镜对对应激光先扩束再聚焦的设计 ${ }^{[30]}$.

微射流水导激光的传输效率是一个关系到加工效 率的复杂关键问题, 其传递效率受激光与水射流的耦合 精度、激光光束能量分布、激光模式以及水层厚度、 射流稳定性影响. 其中, 耦合装置的设计是关键, 其所需 考虑相关因素包括: 水层的厚度、入射角度、速度、 层流、紊流的具体形态、喷嘴的形状形貌、材质.
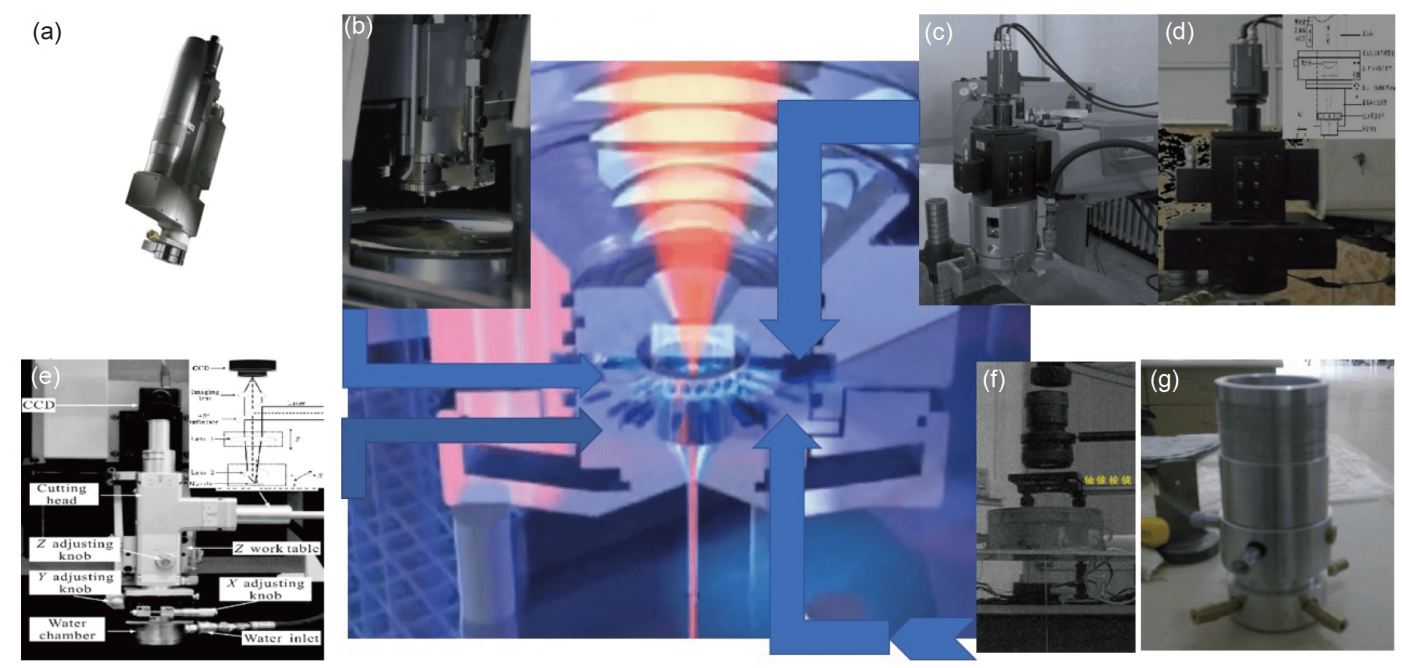

图 3 (网络版彩图)典型微射流水导激光激光水射流耦合头装置. (a) Synova公司切割耦合激光头; (b) Synova公司的晶圆切割; (c), (d) 哈尔滨工业大学两套典型耦合装置 ${ }^{[8,11]}$; (e) 清华大学三轴精细可调激光耦合装置 ${ }^{[26]}$; (f) 带轴雉棱镜的水导激光耦合装 置 ${ }^{[25]} ;(\mathrm{g})$ 带倒置望远镜聚焦微射流水导激光耦合装置 ${ }^{[30]}$

Figure 3 (Color online) Scheme of laser microjet, laser total internal reflect and waveguided into microjet. (a) Laser coupling instrument invented by Synova; (b) coupling instrument of wafer dicing developed by Synova; (c), (d) coupling instrument developed by HIT [8,11]; (e) off-axis coupling instrument developed by Han's team from Tsinghua University [26]; (f) coupling instrument with axicon non-diffracting lens [25]; (g) coupling instrument for LMJ with inverted telescope optical system [30]. 


\section{1 耦合腔体装置设计影响激光耦合效率}

加工激光经过耦合水层自腔体下端聚焦入射，然 后于喷嘴内聚焦随水射流全反射传输, 传输过程需克 服拉曼散射 ${ }^{[31]}$ 、布里渊受激散射. 经过容纳水射流喷 嘴之前过渡的腔体, 入射激光的位置越靠喷嘴, 光强 的强度越大，则受拉曼散射影响激光能量损耗越严重， 水层越厚, 不同加工激光的损耗越多.

传输过程，激光的能量密度经由理想自由伯努利 方程运动射流轴心以及外层水束光纤, 根据Beer-Lambert定律, 即

$I_{x}=I_{0} \exp (-\beta x)$,

其中 $I_{x}$ 为激光功率密度, 增加激光脉宽或水束直径都 导致激光功率密度的减小, 这是激光能量密度衰减的 主要原因, 表 2 所示为沿喷嘴出射方向, 距离喷口不同 位置的激光能量分布所测得结果 ${ }^{[1,16]}$.

喷嘴的材料也从图3(c)铜喷嘴到耐磨性好的图 $3(\mathrm{~g})$ (工具显微镜放大)的(适装腔体)红宝石喷嘴、金刚石喷 嘴、ROTEC材料等, 将来, 还将由于加工能力的提高 和新型材料的出现而不断获得改进. 由于加工的铜喷 嘴(电火花加工)受射流磨损以及待传输的高功率加工 激光的照射影响, 在耦合操作上若有不慎, 就达不到所
要的加工精度; 若采用传统的红宝石拉丝工艺在宝石 镶嵌材料上加工微孔，典型的红宝石元件厚度达到 $0.3 \mathrm{~mm}$, 外圆直径 $\varphi 1.5 \mathrm{~mm}$, 通孔直径为 $\varphi 100 \mu \mathrm{m}$, 雉 形槽雉角 $120^{\circ}$, 深径比 $L_{\mathrm{Noz}} / d_{\mathrm{Noz}} \approx 1.5$, 喷孔入口边缘轮 廓清晰，典型制造的金刚石喷嘴及耦合水腔体结构如 图4所示.

综合考虑激光传输受水射流的影响；2004年， Kruusing ${ }^{[32,33]}$ 指出加工激光经过越薄的耦合水层厚度 入射, 其激光传输效率越高. 受限于实际设计需求, 水 层太薄, 激光容易打坏上方的透明元件 ${ }^{[12]}$, 道理实际 同一些激光诱导等离子体辅助加工一致, 激光入射的 耦合水层厚度应该约为 $2 \mathrm{~mm}$ 较为合适 ${ }^{[12]}$.

\section{2 射流稳定影响微射流水导激光加工}

稳定微水射流可以使得参与加工的激光全反射传 输于多模水束光纤射流内, 并引导激光至工件待加工 表面，同时冲刷加工后的熔渣甚至重铸层，并持续柔 性延伸加工激光的焦点. 由此可见, 水束光纤对于微 射流水导激光系统稳定工作的重要意义，常见的微射 流水导水束光纤直径为20-200 $\mu \mathrm{m}$. Ng Eddie和Guan$\mathrm{nan}^{[34]}$ 则对 $30 \mu \mathrm{m}$ 水束光纤稳定传输激光的穿透效率 和稳定工作距离进行仿真和相关实验; 李灵、李春奇

表 2 耦合微射流激光在空气中传输的能量分布比较 ${ }^{[8,20]}$

Table 2 Tables of laser distribution and beam profile $[8,20]$

\begin{tabular}{|c|c|c|}
\hline 激光能量分布 & 优化前 & 优化后 \\
\hline \multirow[t]{2}{*}{$\begin{array}{l}\text { 激光能量分布 } \\
\text { 受NA影响 }\end{array}$} & & \\
\hline & $\mathrm{NA}=0.177, D=130 \mu \mathrm{m}, W_{\mathrm{p}}=4 \mathrm{MPa}, W_{\mathrm{d}}=40 \mathrm{~mm}$ & $\mathrm{NA}=0.05677, D=180 \mu \mathrm{m}, W_{\mathrm{p}}=4 \mathrm{MPa}, W_{\mathrm{d}}=40 \mathrm{~mm}$ \\
\hline $\begin{array}{c}\text { 激光能量分布 } \\
\text { 受距离焦点沿轴向距离影响 }{ }^{[1]}\end{array}$ & 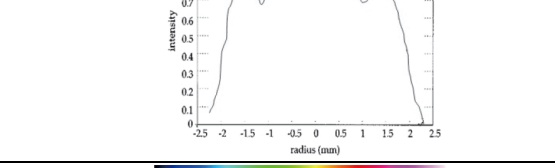 & 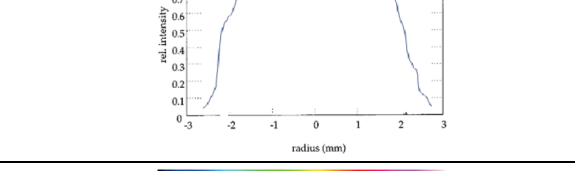 \\
\hline $\begin{array}{l}\text { 激光能量分布 } \\
\text { 受射流影响 }{ }^{200]}\end{array}$ & & \\
\hline
\end{tabular}




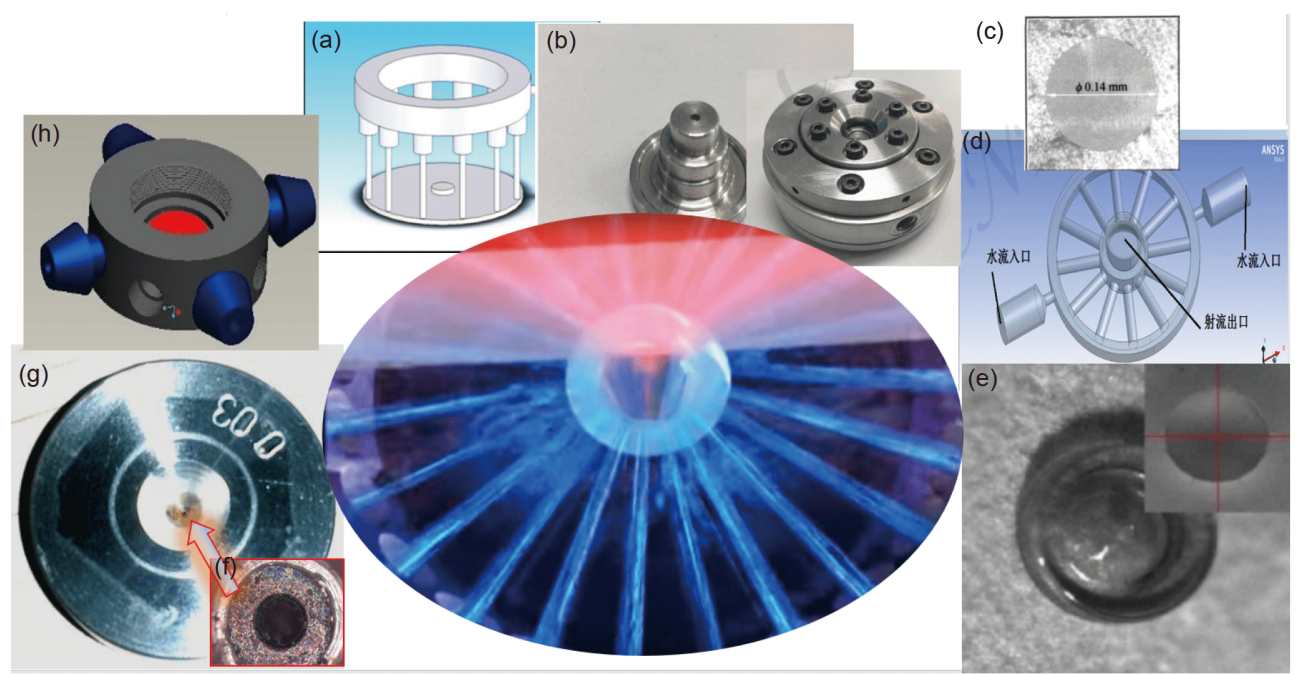

图 4 (网络版彩图)微射流水导激光典型激光水射流耦合腔体及前端喷嘴. (a) 上端送水, 底部喷嘴进水的水腔体 ${ }^{[10]}$; (b), (c) $0.14 \mathrm{~mm}$ 铜喷嘴 ${ }^{[10]}$; (d) 同层水腔体结构; (e) 装配的蓝宝石喷嘴 ${ }^{[16]}$; (f)-(h) $0.03 \mathrm{~mm}$ 红宝石喷嘴 ${ }^{[30]}$

Figure 4 (Color online) Structure of laser microjet nozzle and coupling chamber. (a) Chamber with upside inlet and low outlet [10]; (b), (c) $0.14 \mathrm{~mm}$ copper nozzle [10]; (d) the nozzle in the same level; (e) sapphire nozzles [16]; (f)-(h) the $0.03 \mathrm{~mm}$ diameter ruby nozzle [30].

团队 ${ }^{[8,11]}$ 分析射流的入射角度和压力, 射流测试的压力 范围为5-50和 2-4 MPa (高压小流量的微射流), 这些参 数要求提高了水束光纤发生装置的设计和制造的难 度; 詹才娟团队 ${ }^{[35]}$ 则分析了微射流水导激光的散热效 率提出相关仿真模型. 产生稳定水束光纤需满足的腔 体及喷嘴条件如下: 喷嘴的深径比要求约 $>10$; 水射流 出射要求稳定, 要求避免出现紊流现象, 否则会影响到 激光内部全反射传输; 水射流经过的喷嘴内表面要平 滑光洁, 使得产生的雷诺系数扰流效果减低(故而喷嘴 的内壁加工质量要高), 异形喷嘴的稳定射流效果不显 著, 确保水束内的光线在水束与空气界面发生全反射, 并保证高的全反射效率; 水质要求纯度高、杂质少, 有 利于水束光纤的稳定和减小激光与水的相互作用; 水 束截面圆整, 保证射流稳定性. 本文对水束光纤的特 征进行分析讨论, 研究设计了一套水束光纤发生系统, 包括射流喷孔元件、射流装置和供水系统.

微射流水导激光加工研究中对生成的水束光纤传 导激光的长度进行检测. 水射流导引激光加工能提高 激光加工的焦深, 在此之前, 无论是水辅激光加工 ${ }^{[36]}$ 还是传统激光都使得激光的轴向加工范围上有太大的 限制, 而水射流导引激光一方面对于加工的熔渣进行 冲洗，使得加工后的热熔渣、毛刺等激光加工断面遮 蔽物减少，即使得射流内部全反射的加工激光再次聚 焦加工于延伸焦点的激光, 减少加工表面的熔后残渣
对加工的不利影响; 同时, 微射流水导激光的加工深 度取决于激光传输于水射流的稳定长度, 如图 5 所示, 实验研究结果表明, 水射流稳定性主要受如下因素 影响.

射流喷嘴的加工质量: 包括加工喷嘴的长径比、 孔壁的倾斜度、粗敉度、喷嘴及腔体的装配同轴度 等. 射流㫤的参数及稳定性: 包括射流喷洜的工作方式 (浴轮、柱塞、齿轮等)、初速度、射流压力对于稳定 传输激光射流长度的影响等.

为了获得出离喷嘴稳定水压的自由射流, 微射流 水导激光耦合装置采用如图4(b)所示轴对称的方式入 水, 水射流从两侧进入经由阶梯上升的设计沿上方圆 周对称布置约偶数个孔注入, 中间微孔射出, 此处传 导激光的水束光纤应以稳定射流层流为主, 轴对称入 口的设计可尽量保证耦合装置内水压的对称稳定性. 经过仿真和计算, 研究发现入射的射流喷嘴越接近出 射喷嘴的尺寸, 射流越稳定, 越容易形成喷嘴深度较 大的长稳定自由射流; 入射喷嘴角度越平缓越好, 喷 嘴外沿可以采用 $41^{\circ}$ 过渡角 ${ }^{[37,38]}$, 进入过渡角前的一阶 可以利用优化角度过渡进入喷嘴, 否则容易引起压力 波动, 产生紊流 ${ }^{[39]}$.

\section{3 散射影响激光耦合传输效率}

在水射流距离喷嘴较远时, 相对于功率密度小的 


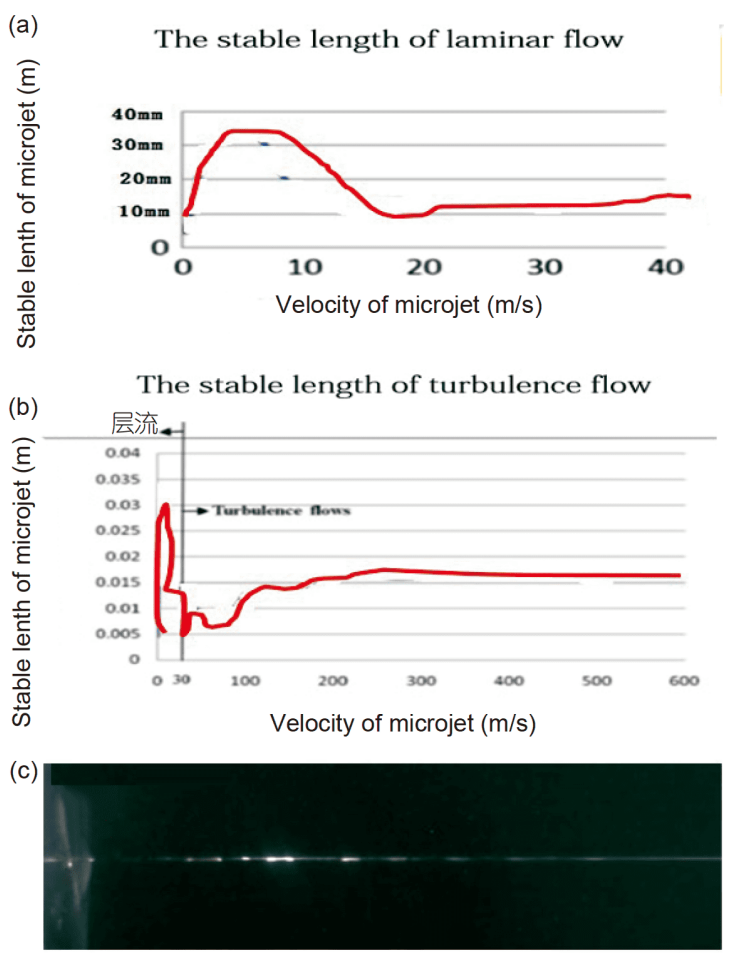

图 5 (网络版彩图)微射流水导激光典型激光水射流稳定传 输长度. (a) 稳定层流长度; (b) 喷嘴对应的湍流长度; (c) 实测 导光射流长度 ${ }^{[8]}$

Figure 5 (Color online) Stable length of laser microjet (LMJ) water. (a) Stable of laminar flow with different nozzle; (b) stable of turbulence flow with different nozzle; (c) measure the stable length of microjet fiber [8].

激光，功率密度大的激光会产生更强的非线性受激拉 曼散射现象，模式越多的激光传输效率越高. 选择合 适的工作距离, 水束光纤入射角度如表2所示, 从而指 导了激光的孔径比、水导激光横模、水射流工作长 度、腔体内对准耦合光加工激光功率参数的选择. 目 前，该系统相关设计中，以下影响因素是设计的关键: 激光在喷嘴水射流中的聚焦位置(径向、轴向)、激光 与微水射流耦合的横向(径向)耦合精度、轴向耦合精 度等. 设计合理的结构, 使得激光于尽可能浅的水层处 耦合入射. 精确聚焦的加工激光即可以优化高效传输 加工激光, 并获得尽可能均匀传输的加工激光.

如表2以及公式(1)Beer-Lambert定律所示, 激光传 输横模数值孔径越小, 射流直径越大, 耦合光斑越细 碎, 并且传输分布越均匀. 激光的脉冲宽度增加时, 获 得的激光加工单脉冲能量更大，直接关系到激光传输 的效率.

\section{4 水射流导引激光加工改善加工表面质量}

微射流水导激光加工时, 射流场作为辅助能场, 产 生机械力, 其冲击加工凹槽底部的反作用力, 使得射流 能带走激光加工形成的熔渣、残渣、粉尘、颗粒甚至 毛刺. Rashed等人 ${ }^{[40]}$ 对比了微射流水导激光切割和微 细电火花加工不锈钢的切槽表面质量, 哈尔滨工业大 学的王扬、杨立军两位教授及李春奇博士团队 ${ }^{[40-42]}$ 则是详细描述了 $\mathrm{TC} 4^{[43]}$ 、镁合金等的切槽和打孔等的 加工表面质量的改善, 表明射流同时能产生冷却作用. 激光在水射流中加工 $\mathrm{Si}^{[44]}$ 的仿真, 实际加工也有相关 阐述.

激光加工的汽化、残渣、颗粒遇纯净水后, 冷却 并凝结成粉末颗粒，并能够及时随射流冲刷并离开已 经发生相变的旧加工断面. 去离子微水射流在冲刷和 撞击激光切出的槽型时, 能够成功改善被加工断面的 组织, 使之更细淢, 并且更有效地改善激光加工精度, 减小加工锥度. 目前, 金属材料、光电材料 $\left(\mathrm{Si}^{[44]}\right.$, $\mathrm{SiC}^{[45]}, \mathrm{GaN}^{[13]}$ 等)及低 $k$ 材料的加工都试图利用微射流 水导激光作为一种提高纵向及横向加工效果的手段.

\section{1 均衡径向、轴向加工能力}

相对常规激光的高斯分布而言, 微射流水导激光 的加工特点同激光的能量分布有直接关联, 其待加工 工件具有更好的垂直面加工质量以及更高的径向切割 效率, 因此特别适合切缝及钻孔.

\section{2 降低加工热, 减少热影响区}

微射流水导能够不断流动以更新热传导层. 加工 时需要控制水下激光的峰值能量密度. 若高能脉冲激 光束辐照到材料上造成材料热堆积破坏, 汽化的蒸气 粒子, 包括更高脉冲能量阈值激发的等离子体、残 渣、高压等离子体和蒸气迅速膨胀形成冲击波. 但去 离子水、溶液(硫酸铜)的存在都会限制等离子体膨胀, 从而造成对工件的反作用力，并对加工区域的热影响 区或者残渣起到力学剥除的影响, 此即为等离子体冲 击波作用力. 研究表明冲击波反作用力在切割蓝宝石 时也较为有效 ${ }^{[46,47]}$.

无论是水射流的水层流动，还是等离子体带动的 冲击波及所产生的蒸气都可改善加工表面, 分散加工 多余能量, 减少热堆积, 限缩热影响区, 以 $\mathrm{SiC}$ 为例的 
加工效果如图6(a)和(b)所示 ${ }^{[48]}$; 图6(e)为无水激光加工 的电镜图, 图6(f)为有水激光加工的电镜图 ${ }^{[49]}$. 无水冷 却的激光加工槽型内槽更细小, 锥度更大, 边缘更粗 粘; 而有水冷却的激光加工有效加工内槽更宽大，锥 度更小, 另外断面组织也更细小. 此时, 可以看到激光 加工时冲击波的机械冲击力发生作用. 另外, 以氮化硅 为例，水中激光功率决定微射流水导激光的加工能力, 但同激光加工不同的是，LMJ作用于工件表面的雉度 等形貌参数, 不只受能量密度影响, 也受激光的峰值 功率、激光的脉冲作用时间、频率等与水射流相互作 用的参数 ${ }^{[50]}$ 影响.
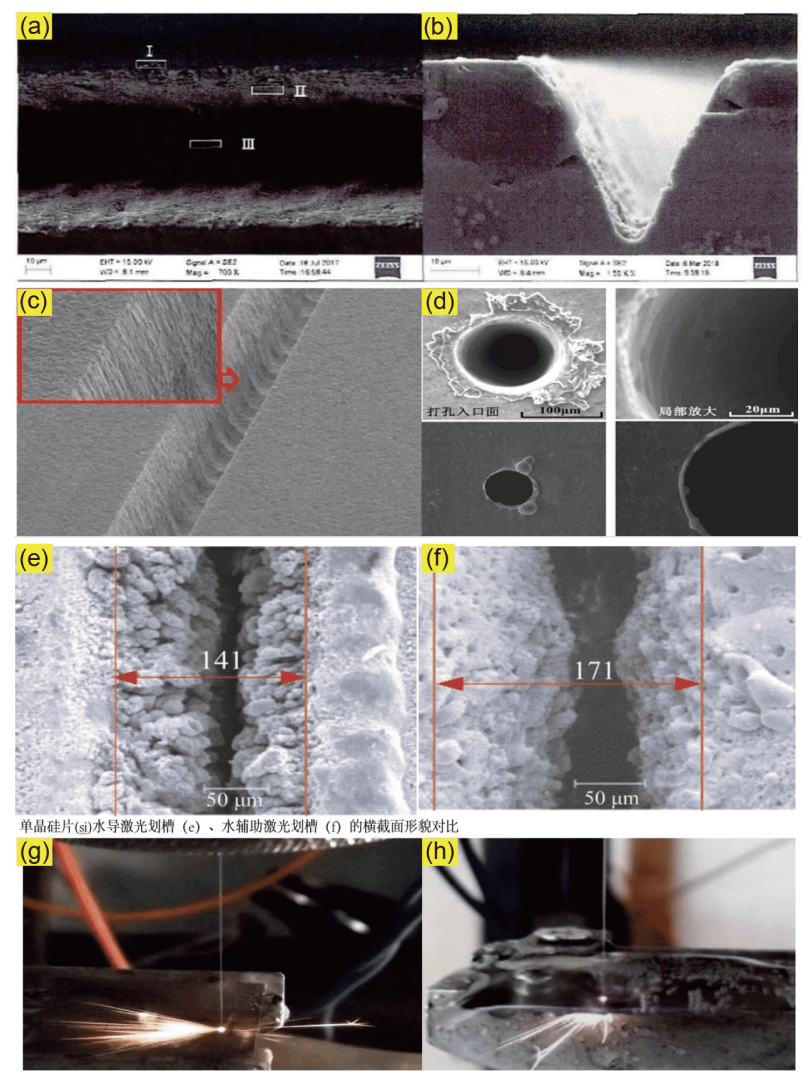

图 6 (网络版彩图)稳定微射流水导激光典型加工改善径向 加工能力并改善表面质量 ${ }^{[8]}$. (a), (b) $4 \mathrm{H}-\mathrm{SiC}$ 切割横断面扫描 电镜图 ${ }^{[48]}$; (c), (d) 微射流水导激光加工硅的出入口质量图; (e), (f) $\mathrm{Si}_{3} \mathrm{~N}_{4}$ 激光及水导激光加工的扫描电镜图 ${ }^{[49]} ;(\mathrm{g}),(\mathrm{h})$ 水导激光加工TC4金属薄板 ${ }^{[8]}$

Figure 6 (Color online) Enhancement of cross section processing quality through stable laser microjet (LMJ) processing [8]. (a), (b) The cross section SEM pictures of $4 \mathrm{H}-\mathrm{SiC}$ cutting with laser cutting assisted with microjet [48]; (c), (d) the different qualities between outlet and inlet drilled by LMJ; (e), (f) the SEM pictures which show the different $\mathrm{Si}_{3} \mathrm{~N}_{4}$ cross section quality processed by laser and LMJ with vibration [49]; (g), (h) LMJ process thin TC4 metal alloys [8].
与此同时, 水射流可改善激光加工的质量, 以 $\mathrm{Si}$ 为 例, 也可以从图6(a)和(b)看出. 由于激光的能量峰值下 降, 故而对于待加工工件的径向加工能力来说是下降 了. 这一点也可以从图6(e)和(f)看出, 微射流水导激光 无法提高加工速度, 但是可以改善激光加工质量, 也可 以切透更厚的TC4(Ti6Al4V)样品工件.

\section{3 加工对象选择加工激光}

在微射流水导激光中仍然需要根据待加工材料对 相应波长, 脉宽激光吸收效率选择激光. 譬如氮化 硅 ${ }^{[49]}$ 可选用大功率 $1064 \mathrm{~nm}$ 的 Nd:YAG激光器进行加 工，高反射率的抛光金属紫铜，电路板铜管脚 ${ }^{[50]}$ 也可 以, 硅 ${ }^{[51]}$ 甚至可以选择准分子激光器进行加工, 铝等 高反材质金属可以选择各自不同参数的激光器进行加 工 ${ }^{[52,53]}$. 由于微射流水导激光削弱了激光的峰值能量, 所以使得该技术在切割高反射率材料上加工效率的优 势不明显.

LED相关光电材料 ${ }^{[54]}$ 以及低介电材料, 如 $\mathrm{PVD}^{[55]}$, $\mathrm{CVD}$ 等金刚石材料适合光子能量更大，穿透能力更强 的532 nm波长激光耦合水射流加工.

\section{5 微水射流导引激光工业应用}

随着大规模利用传统激光甚至短脉冲激光在大多 数材料上钻孔、切缝、切槽加工，微射流水导激光由 于是常规激光器的复合加工, 在一些需要大深度钻 孔、切割、切缝上仍然有望得到推广. 目前, 在航空 发动机上的气膜孔加工方面特别有望得到应用, 其他 方面的扩展也得到持续关注和研究, 有望得到推广.

\section{1 微射流水导激光加工低 $k$ 绝缘介质}

电路板上的大量低 $k$ 绝缘介质层需要钻孔, 由于绝 缘体表面上自由电子较少，故而加工时沿温度梯度较 低、散热良好的朝向进行加工，微射流水导激光则可 以控制热量的扩散，该技术甚至可以和电解液射流耦 合, 实现更高质量的激光电解切槽、钻孔 ${ }^{[56]}$. 其参考 模型则包括树脂、塑料等溶沸点较低的材料, 主要靠 激光热量使其发生相态变化.

该加工方式可以提高激光的加工质量和加工速 度, 却无法加工值更高的难加工材质. 优良的散热性能 使得该加工方式也适合加工绝缘体(低 $k$ 材料). 
该加工方式除了加工范围更广以外，也对于加工 后的雉角有所控制, 通常切透的背面孔都会大于正面 切缝.

\section{2 加工硬质合金}

该加工方式对于受热敏感硬质合金的加工也很有 益处. 例如，该加工方式可以应用于心脏支架的加工， 也可用于加工精密合金刀具前后刃角的凹槽结构. 同 时, 微射流水导激光加工方式对于抛光过的高反射率 加工作用无法得到显现. 对于激光吸收效率低的材料 (譬如高反射率纯铜, 低吸收率的铝等材料), 微射流水 导激光无法提高加工速度, 只能有限地控制裂纹生长 并改善加工.

\section{3 光电材料的切割和缝槽等的加工}

微射流水导激光切槽切割加工常见的对象包含通 常比较硬而脆的光电材料, 形状复杂的 Si如金刚石 $(\mathrm{PCD})$ 、氮化硅、立方氮化硼 ${ }^{[57]}$ 、蓝宝石 ${ }^{[58]}$ 等. $10 \mathrm{~W}$ 的脉冲 $532 \mathrm{~nm}$ 绿光激光耦合微射流水导激光也可以用 于加工金刚石; $23 \mu \mathrm{m}$ 射流的侧向微射流水导激光可 在 $4 \mathrm{H}-\mathrm{SiC}$ 上加工 $25 \mu \mathrm{m}$ 切缝.

\section{6 微水射流导引激光其他应用}

微射流水导激光在激光加工深孔上有着不可忽视 的性价比, 除了可以实现大厚度的激光切缝, 打孔以 外, 优良的散热性能使得该加工方式也可用于木材板 块零件加工 ${ }^{[59]}$; 针对于加工阈值低, 吸收效率高的工 业应用场合, 甚至于譬如阵列式的透镜组耦合一些阵 列式低压水射流喷嘴, 进行生物组织表面的表面织 构 ${ }^{[60,61]}$ 等, 如图7(b)所示.

\section{7 微水射流导引激光及研究展望}

微射流水导激光在激光加工机理上仍可认为是传

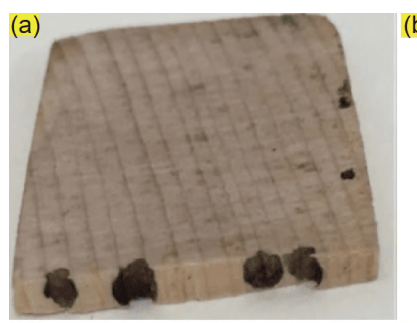

(b)

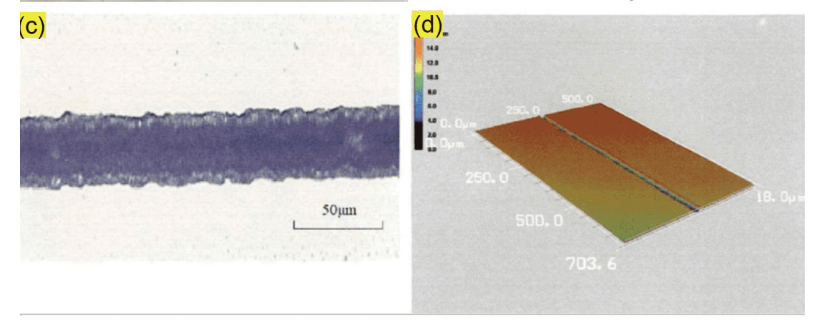

图 7 (网络版彩图)LMJ的应用前景及展望. (a) 水导激光加 工带一定烧蚀痕迹的木板 ${ }^{[59]}$; (b) 带微透镜阵列的低压水射 流喷嘴作用于皮肤去斑点; (c), (d) 加工一些改善边缘质量的 $\mathrm{SiC}$ 的显微图及3D形貌扫描图

Figure 7 (Color online) (a) Laser micro processing on wood [59]; (b) LMJ coupled with micro lens array on skin; (c), (d) the profile processed with LMJ on SiC.

统脉冲、短脉冲激光热加工的机理, 不同的射流耦合 (聚焦点)、流速、孔径等是控制激光产生加工热的不 同变量因素.

微射流水导激光区别于飞秒激光深孔加工 ${ }^{[62]}$, 该 加工方式可在提升传统激光加工质量、加工工业深孔 有更广大更灵活的应用前景. 比如, 高附加值的切槽要 求的各种手术刀具、支架、半导体基底场合，这些应 用主要考虑实际加工及性价比. 除了可实现强度大、 切口深、大厚度的激光切缝、打孔以外, 该技术可控 制一定传统激光加工热量, 虽不具备飞秒激光高功率 峰值实现绝对冷加工的能力, 但同样可以在一定程度 上获得更高质量的 $10 \mu \mathrm{m}$ 级的缝、槽、孔. 因此, 该技 术可以使得原有的激光器发挥更大的作用, 也可以提 高原有传统激光的加工质量和加工速度.

总之, 在提高传统激光加工效率、质量、厚度方 面, 微射流水导激光仍然可以有其较为广泛的微加工 应用价值.

\section{参考文献}

1 Richerzhagen B, Delacretaz G P, Salathe R P. Complete model to simulate the thermal defocusing of a laser beam focused in water. Opt Eng, 
1996, 35: 2058-2067

2 Richerzhagen B. Method and apparatus for machining material with a liquid-guided laser beam. US Patent, 5902499, 1999-05-11

3 Marimuthu S, Dunleavey J, Liu Y, et al. Water-jet guided laser drilling of SiC reinforced aluminium metal matrix composites. J Compos Mater, 2019, 53: $1-10$

4 Mai T A, Richerzhagen B, Snowdon P C, et al. The Laser MicroJet (LMJ): A multi-solution technology for high quality micro-machining. In: Proceeding of the SPIE-The International Society for Optical Engineering, Laser-based Micro and Nano packaging and Assembly. San Jose, 2007. 6459: 64590P

5 Couty P, Wagner F R, Hoffmann P W. Laser coupling with a multimode water-jet waveguide. Opt Eng, 2005, 44: 068001

6 Romolia L, Lovicua G, Rasheda C A A, et al. Microstructural changes induced by ultrashort pulsed lasers in microdrilling of fuel nozzles. In: Proceeding of the 9th CIRP Conference on Intelligent Computation in Manufacturing Engineering. Napoli, 2015. 33: 509-514

7 Bruckert F, Pilat E, Piron P, et al. Tailor cutting of crystalline solar cells by laser micro jet. In: Proceedings of SPIE-The International Society for Optical Engineering, San Francisco, 2012. 8239: 82390P

8 Li C Q. Research on Water-Jet Guided Laser Micromachining System and Key Technology (in Chinese). Dissertation for Doctoral Degree. Harbin: Harbin Institute of Technology, 2012 [李春奇. 水导激光微细加工系统及关键技术研究. 博士学位论文. 哈尔滨: 哈尔滨工业大学, 2012]

9 Hopman S, Fell A, Mayer K, et al. Comparison of laser chemical processing and laser microjet for structuring and cutting silicon substrates. Appl Phys A Mater, 2009, 95: 857-866

10 Sun B Y, Qiao H C, Zhao J B, et al. Current status of water-jet guided laser cutting technology (in Chinese). Opt Electron Eng, 2017, 44: 10391128 [孙博宇, 乔红超, 赵吉宾, 等. 水导激光切割技术研究现状. 光电工程, 2017, 44: 1039-1128]

11 Li L. Study on Water-Jet Guided Laser Micromachining Technology (in Chinese). Dissertation for Doctoral Degree. Harbin: Harbin Institute of Technology, 2008 [李灵. 水导激光微细加工技术研究. 博士学位论文. 哈尔滨: 哈尔滨工业大学, 2008]

12 Richmann A, Kurzen S, Carron B, et al. Cutting diamond tools using the laser microjet technology on a 5-axis machine. In: Proceeding of the Lasers in Manufacturing Conference-LIM2015. Munich, 2015. 100

13 Wang H Z. The technology principle and application of water-jet-guided laser scribing (in Chinese). Equipment Electron Products Manuf, 2008, 3: 27-31 [王宏智. 微水导激光划片工艺原理及应用. 电子工业专用设备, 2008, 3: 27-31]

14 Wagner F, Sibailly O, Vago N. The Laser Microjet Technology - 10 Years of Development. In: Proceedings of the International Congress on Applications of Lasers \& Electro-Optics. Ecublens, 2003

15 Zhou R, Li F P, Hong M H. Laser interaction with materials and its applications in precision engineering (in Chinese). Sci Sin-Phys Mech Astron, 2017, 47: 024201 [周锐, 李峰平, 洪明辉. 激光与物质相互作用及其精密工程应用. 中国科学: 物理学 力学 天文学, 2017, 47: 024201]

16 Chen X. Studies on Mechanisms of the Interaction Between High-Power Laser and Matter in Water (in Chinese). Dissertation for Doctoral Degree. Nanjing: Nanjing University of Science \& Technology, 2004 [陈笑. 高功率激光与水下物质相互作用过程与机理研究. 博士学位论 文. 南京: 南京理工大学, 2004]

17 Nesterov A V, Niziev V G, Yakunin V P. Generation of high-power radially polarized beam. J Phys D-Appl Phys, 1999, 32: $2871-2875$

18 Liu X, Long Y H, Bao J D, et al. Investigation of water flow characteristics based on water assisted laser processing (in Chinese). Laser Tech, 2017, 41: 442-446 [刘金金, 龙芋宏, 鲍家定, 等. 基于水辅助激光加工的水层流动特性的研究. 激光技术, 2017, 41: 442-446]

19 Long J, Weng Q, Hong W, et al. Fast water flow in laser micromachined microgrooves with nonuniform surface wettability. Exp Thermal Fluid Sci, 2019, 103: 9-17

20 Lu X Z, Jiang K Y, Jiang F, et al. Laser microjet enhanced processing energy distribution (in Chinese). Chin App Laser, 2015, 35: 230-235 [卢希 钊, 江开勇, 姜峰, 等. 微射流水导改善加工激光能量分布. 应用激光, 2015, 35: 230-235]

21 Mai T A, Richerzhagen B. New process for cutting of 4th generation OLED masks: Laser microJet technology. In: Proceedings of the Fourth International WLT-Conference on Lasers in Manufacturing. Stuttgart, 2007. 747-750

22 Yang L J, Kong X J, Wang Y, et al. Laser micro-holes machining technology and its application. Aeronaut Manuf Tech F, 2016, 19: 32-38 [杨立 军, 孔宪俊, 王扬, 等. 激光微孔加工技术及应用. 航空制造技术, 2016, 19: 32-38]

23 Zhan C J, Li C F, Pan Y C, et al. Dynamical stress reliability and sensitivity analysis of nonlinear rotor system with rigid-flexible structure (in Chinese). Chin Quarter Mech, 2011, 32: 159-165 [詹才娟, 李昌烽, 潘永琛, 等. 微水射流导引激光精密打孔过程的流动分析. 力学季刊, 2011, 32: 159-165] 
24 Mullick S, Madhukar Y K, Roy S, et al. Development and parametric study of a water-jet assisted underwater laser cutting process. Int J Mach Tool Manuf, 2013, 68: 48-55

25 Ye R F, Shen Y, Wang L, et al. Novel coupling system of water-jet guided laser (in Chinese). J Xiamen Univ, 2009, 48: 369-372 [叶瑞芳, 沈阳, 王否, 等. 新型水导引激光耦合系统研究. 厦门大学学报, 2009, 48: 369-372]

26 Sun D, Wang J H, Han F Z. Research on coupling technology for water-jet guided laser machining based on off-axis optical system (in Chinese). Infrared Laser Eng, 2018, 47: 1206001 [孙冬, 王军华, 韩福柱. 基于离轴光学系统的水导激光耦合技术研究. 红外与激光工程, 2018, 47: 1206001]

27 Li L, Yang L J, Wang Y, et al. Laser and water-jet fiber coupling technology for water-jet guided laser micromachining (in Chinese). Opt Precision Eng, 2008, 16: 1615-1621 [李灵, 杨立军, 王扬, 等. 水导激光微细加工中激光与水束光纤耦合技术. 光学精密工程, 2008, 16: $1615-1621$

28 Li M, Zhang W W, Zhang X P, et al. A simple optical coupling method for water-jet guided laser machining system. Appl Mech Mater, 2014, 541-542: 774-779

29 Yang B J, Xie S D, Dai F. Design of coupling device for laser cutting guided by water beam (in Chinese). Laser Tech, 2017, 41: 247-250 [杨保 健, 谢顺德, 代福. 微水导激光切割玻璃的耦合装置设计. 激光技术, 2017, 41: 247-250]

30 Lu X Z. Researching Key Technology of Gravure Engraving Based on Stable Micro-Jet Waveguide Laser Processing (in Chinese). Dissertation for Doctoral Degree. Xiamen: Xiamen University, 2011 [卢希钊. 稳定微射流水波导激光直刻凹印制版关键技术研究. 博士学位论文. 厦门: 厦门大学, 2011]

31 Spiegel A, Vago N, Wagner F R. High efficiency Raman scattering in micrometer-sized water jets. Opt Eng, 2004, 43: 450-454

32 Kruusing A. Underwater and water-assisted laser processing: Part 1 - General features, steam cleaning and shock processing. Opt Lasers Eng, 2004, 41: 307-327

33 Kruusing A. Underwater and water-assisted laser processing: Part 2-Etching, cutting and rarely used methods. Opt Lasers Eng, 2004, 41: 329352

34 Ng Eddie Y K, Guannan D. The stability of 30- $\mu$ m-diameter water jet for jet-guided laser machining. Int J Adv Manuf Technol, 2015, 78: 939946

35 Zhan C J, Li C F, Wang R, et al. Analyses of heat transfer in micro-waterjet guided laser drilling process (in Chinese). J Eng Thermophys, 2011, 32: 111-114 [詹才娟, 李昌烽, 王瑞, 等. 微水射流导引激光打孔的传热分析. 工程热物理学报, 2011, 32: 111-114]

36 Mai T A, Kling N U, Vago N, et al. Laser MicroJet ${ }^{\circledR}$ Technology_Fundamental Study and Highlights of It's Latest Application, Advanced Laser Applications Conference, ALAC 2007. San Jose, 2007

37 Yuan G F, Yao Y S, Chen X H. Experimental study on the quality of material surface applied laser-chemical combined etching (in Chinese). Chin J Laser, 2010, 37: 281-283 [袁根福, 姚燕生, 陈雪辉. 激光和化学复合刻蚀加工表面质量的实验研究. 中国激光, 2010, 37: 281-283]

38 Fu Y H, Cao J, Dong F, et al. CFD simulation of the laser stable water beam in micro water-jet guided laser (in Chinese). Fluid Mach, 2013, 41: 21-25 [符永宏, 曹佳, 董非, 等. 微水导激光稳定水束光纤的CFD仿真研究. 流体机械, 2013, 41: 21-25]

39 Yang Y S, Zhang J P, Nie S L. Energy loss of nozzles in water jet system (in Chinese). J Mech Eng, 2013, 49: 139-145 [杨友胜, 张建平, 聂松林. 水射流喷嘴能量损失研究. 机械工程学报. 2013, 49: 139-145]

40 Rashed C A A, Romoli L, Tantussi F, et al. Water jet guided laser as an alternative to EDM for micro-drilling of fuel injector nozzles: A comparison of machined surfaces. J Manuf Processes, 2013, 15: 524-532

41 Li C Q, Yang L J, Wang Y. Experimental study on water-jet guided laser machining magnesium alloy. Mater Sci Forum, 2012, 723: 476-480

42 Li C Q, Yang L J, Wang Y. A research on surface morphology of cutting of titanium sheet with water-jet guided laser and conventional laser. Appl Mech Mater, 2012, 120: 366-370

43 Li C Q, Yang L J, Wang Y. Investigation on water-jet guided laser cutting of titanium alloy thin sheets. Energy Educat Sci Tech Part A: Energy Sci Res, 2012, 30: 481-488

44 Li C F, Johnson D B, Kovacevic R. Modeling of waterjet guided laser grooving of silicon. Int J Mach Tools Manuf, 2003, 43: 925-936 Tan S N. Research on Water-Jet Guided Laser Micromachining Technology of SiCp/Al (in Chinese). Dissertation for Master's Degree. Harbin: Harbin Institute of Technology, 2014 [谭淞年. SiCp/Al复合材料的水导激光加工技术研究. 硕士学位论文. 哈尔滨: 哈尔滨工业大学, 2014]

46 Xie X Z, Hu M F, Chen W F, et al. Cavitation bubble dynamics during laser wet etching of transparent sapphire substrates by $1064 \mathrm{~nm}$ laser irradiation. J Laser Micro Nanoeng, 2013, 8: 259-265 
47 Xie X, Huang X, Jiang W, et al. Three dimensional material removal model of laser-induced backside wet etching of sapphire substrate with $\mathrm{CuSO}_{4}$ solutions. Opt Laser Tech, 2017, 89: 59-68

48 Hui Q Z. Study on Process and Material Removal Mechanism for Micromachining 4H-SiC by Laser Assisted Waterjet (in Chinese). Dissertation for Master's Degree. Jinan: Shandong University, 2018 [惠庆志. 激光辅助水射流加工4H-SiC材料的工艺与去除机理研究. 硕士学位论文. 济 南: 山东大学, 2018]

49 Yao Y S, Chen Q B, Wang J, et al. Water-jet assisted laser precision processing of $\mathrm{Si}_{3} \mathrm{~N}_{4}$ ceramics (in Chinese). Opt Precision Eng, 2018, 26: 2723-2731 [姚燕生, 陈庆波, 汪俊, 等. 氮化硅陶瓷水射流辅助激光精密加工. 光学精密工程, 2018, 26: 2723-2731]

50 Kruusing A. Handbook of Liquids-Assisted Laser Processing. Holland: Elsevier Science Ltd., 2007. 143-203

51 Long Y H, Xiong L C, Shi T L. Experimental research of micromachining silicon by excimer laser ablation in air and under water (in Chinese). Laser Tech, 2006, 30: 567-569 [龙芋宏, 熊良才, 史铁林. 水辅助准分子激光微加工硅的实验研究. 激光技术, 2006, 30: 567-569]

52 Porter J A, Louhisalmi Y A, Karjalainen J A, et al. Cutting thin sheet metal with a water jet guided laser using various cutting distances, feed speeds and angles of incidence. Int J Adv Manuf Technol, 2007, 33: 961-967

53 Liu B. Study of Processing Technology Based on Laser-Microjet (in Chinese). Dissertation for Master's Degree. Harbin: Harbin Institute of Technology, 2012 [刘备. 基于微水导激光加工技术的研究. 硕士学位论文. 哈尔滨: 哈尔滨工业大学, 2012]

54 Perrottet D, Amorosi S, Richerzhagen B. New process for screen cutting: water-jet guided laser. In: Proceedings of SPIE the International Society for Optical Engineering. Society of Photo-Optical Instrumentation Engineers, 2005. 596105

55 Richerzhagen B, Plankensteiner M, Kling N U, et al. Saw+LMJ: A hybrid semiconductor dicing solution. Proc SPIE, 2008, 6880: 1-6

56 Zhang $\mathrm{H}$, Xu J W, Wang J M, et al. Experimental study of hybrid processing of jet electrochemical machining and laser beam machining (in Chinese). Acta Aeronaut Astronaut Sin, 2009, 30: 1138-1143 [张华, 徐家文, 王吉明, 等. 喷射液束电解——激光复合加工工艺试验研究. 航 空学报, 2009, 30: 1138-1143]

57 Melaibari A, Molian P, Shrotriya P. Two-dimensional contour cutting of polycrystalline cubic boron nitride using a novel laser/water jet hybrid process. Int J Adv Manuf Tech, 2012, 63: 641-649

58 Wu Z R. The Mechanism Governing Cutting of Hard Materials with Hybrid Laser/Waterjet System Through Controlled Fracture. Dissertation for Doctoral Degree. Iowa: State University, 2015

59 Jiang X B, Hu H, Liu J Q, et al. Discussion on the processing of wood by nanosecond water guide laser (in Chinese). Sci Silvae Sin, 2018, 54: 121-127 [姜新波, 胡昊, 刘九庆, 等. 纳秒水导激光加工木材工艺探讨. 林业科学, 2018, 54: 121-127]

60 Jang H J, Yeo S, Yoh J J. Skin pre-ablation and laser assisted microjet injection for deep tissue penetration. Lasers Surg Med, 2017, 49: 387-394

61 Kiyama A, Endo N, Kawamoto S, et al. Visualization of penetration of a high-speed focused microjet into gel and animal skin. J Vis, 2019, 22: $449-457$

62 Xia B, Jiang L, Wang S M, et al. Femtosecond drilling micro holes (in Chinese). China Laser, 2013, 40: 1-12 [夏博, 姜澜, 王素梅, 等. 飞秒激光 微孔加工. 中国激光, 2013, 40: 1-12] 


\title{
Research and application development of compound energy field processing — laser microjet
}

\author{
LU XiZhao ${ }^{1,2,3^{*}} \&$ JIANG KaiYong ${ }^{1,3}$ \\ ${ }^{1}$ College of Mechanical Engineering and Automation, Huaqiao University, Xiamen 361021, China; \\ ${ }_{2}^{2}$ Department of Electronic Science, Xiamen University, Xiamen 361005, China; \\ ${ }^{3}$ Fujian Key Laboratory of Special Energy Manufacturing, Huaqiao University, Xiamen 361021, China
}

\begin{abstract}
Laser microjet is a kind of compound energy field processing, which combined laser with microjet processing as well. While processing laser couples with stabilize pressure microjet and transfers internal total reflection until it arrives at workpieces surface, and changes the Gauss energy distribution to flat-topped laser beam, as well as cools and brushes the processing cross section. The stable low flow pressure deionized water jet waveguides cutting laser flexible extension processing focus, to improve the laser energy distribution while removing chips and cooling and enhance the axial processing quality and accuracy, and improves the laser axial processing ability. Talking about the research development of LMJ and the key technologies of LMJ equipment which including the angle of laser total reflect inner microjet, laser quality, laser transverse model, focal points, coupling precision of laser with nozzle and the stable work distance of microjet etc. At the same time, this processing method is introduced which is suitable for controlling the heat of laser processing, enhancing the direction of laser processing, high quality deep holes, slot and seam with large aspect ratio (about 10-100) as well. The object of the processing method gradually includes high value-added occasions of insulating materials, low- $k$ materials, semiconductor, cemented carbide, such as $\mathrm{SiC}$, GaAs, polycrystalline diamond and other photoelectrical materials.
\end{abstract}

laser microjet, coupling, inter total reflect, micro-processing quality

PACS: $42.30 . \mathrm{Sy}, 42.62 . \mathrm{Be}, 47.15 . \mathrm{Fe}, 47.15 . \mathrm{Gf}$

doi: 10.1360/SSPMA-2019-0194 